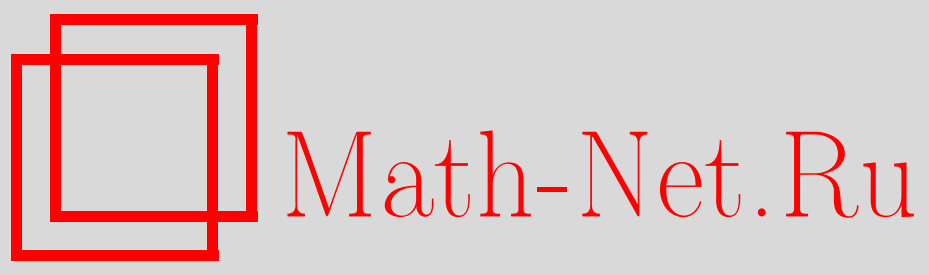

В. В. Воронюк, И. Д. Манджавидзе, А. Н. Сисакян, $S$ матричное описание неравновесных систем с конечной температурой, ТМФ, 2006, том 149, номер 3, 368-380

DOI: https://doi.org/10.4213/tmf5528

Использование Общероссийского математического портала Math-Net.Ru подразумевает, что вы прочитали и согласны с пользовательским соглашением http: //www. mathnet.ru/rus/agreement

Параметры загрузки:

IP : 54.210 .77 .194

26 апреля 2023 г., 18:33:14

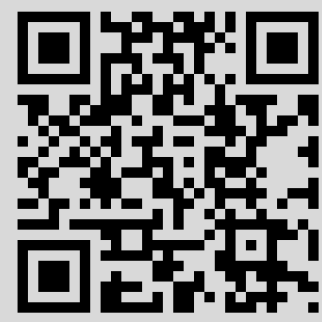




\title{
$S$-МАТРИЧНОЕ ОПИСАНИЕ НЕРАВНОВЕСНЫХ СИСТЕМ С КОНЕЧНОЙ ТЕМПЕРАТУРОЙ
}

\begin{abstract}
Рассматривается “инклюзивный” (“частичный”) метод описания неравновесных диссипативных систем на ранней (кинетической) стадии эволюции, когда распределение температуры неоднородно. Теория возмущений формулируется в терминах локальных в пространстве-времени температурных функций Грина. Получено уравнение Лиувилля для одночастичной функции распределения.
\end{abstract}

Ключевые слова: теория поля в реальном времени при конечных температурах, теория возмущений.

\section{1. ВВЕДЕНИЕ}

Современная концепция сильных взаимодействий основана на предположении, что динамика этих процессов ограничена скрытой неабелевой калибровочной симметрией. Это означает, что в данном случае мы имеем дело с динамикой сильно взаимодействующей системы со скрытыми связями. Почти вековой опыт исследования процессов множественного рождения адронов показывает, насколько сложно найти количественные закономерности в этом случае.

Поэтому разумно начать описание столкновения адронов высокой энергии с максимально упрощенной задачи. Например, можно пренебречь в первом приближении многочастичными корреляциями. В этом заключается “инклюзивный” подход к процессам множественного рождения. Очевидно, что если отбросить многочастичные корреляции, то какая-то информация становится недоступной, скрытой. Но несмотря на это, инклюзивный подход был популярен и широко использовался при исследовании динамики адронов всю вторую половину XX века.

Инклюзивный подход был предложен в ряде работ [1]. В рамках такого приближения исследуются только одно- или, максимум, двухчастичные распределения. Логунов с сотрудниками обнаружили ряд ограничений для одночастичного спектра [2], предсказали их универсальность [3] и пр.

* Объединенный институт ядерных исследований, Дубна, Московская обл., Россия. E-mail: vadimv@nu.jinr.ru; joseph@nusun.jinr.ru; sisakian@jinr.ru 
Существует также статистический метод описания многочастичных систем. Он наиболее эффективен при описании систем, находящихся в равновесии или же близких к нему. Как было предложено в свое время Боголюбовым [4], многочастичные функции распределения "расцепляются" при приближении системы к равновесию на соответствующее произведение одночастичных функций распределения. Иными словами, вблизи равновесия высшие корреляторы должны быть малы. В этом заключается принцип ослабления корреляций Боголюбова.

Тогда естественно предположить, что инклюзивное описание Логунова [1] дает полную картину множественного рождения, если система находится в "равновесии"1). Однако, к сожалению, нет прямой аналогии между статистическим и $S$ матричным описаниями. Дело в том, что в физике частиц понятие "термостата" отсутствует: процесс рождения частиц происходит в вакууме. Поэтому, соответственно, в физике частиц нет обычного для статистической механики определения "равновесного состояния". Мы попытаемся показать необходимую количественную связь между инклюзивным и термодинамическим подходами.

Эта связь состоит в том [5], что возможность инклюзивного описания уже означает малость многочастичных корреляторов. Мы будем говорить [6], что если система достаточно полно описывается средней энергией, средней множественностью и т.д., то система находится в равновесии в том смысле, что для ее описания высшие корреляторы по энергии, множественности и т.д. несущественны. Другими словами, утверждая, что если параметр измерим, т.е. является слабо флуктуирующей величиной, мы имеем равновесие по этому параметру. Такой подход кажется нам интересным, поскольку не требует введения канонического определения понятия "равновесия", основанного на гипотетическом термостате.

Такое описание для однородного распределения температуры было предложено в работе [7]. Замечательно, что если температура является "хорошим" параметром, то этого условия достаточно для восстановления из $S$-матрицы канонической схемы термодинамики.

Этот формализм можно естественным образом расширить также на случай неоднородного распределения температуры. Описание именно этого формализма изложено в данной работе.

Мы разбиваем на ячейки измерителъный прибор, чтобы ввести температуру для каждой отдельной ячейки с 4-координатой $R$ независимо. Здесь важно отметить, что это действительно можно сделать, поскольку в $S$-матричной теории измеряются свободные (на массовой поверхности) частицы, т.е. энергию (и импульс) можно измерить отдельно для каждой ячейки. Заметим, что похожая идея исследовалась в работе [5], где рассматривались ячейки в фазовом пространстве.

Квантовый принцип неопределенности ведет к тому, что 4-размер ячейки $\delta R$ невозможно считать произвольно малым. Это основная трудность рассматриваемого подхода. Нами будет предложено основанное на инклюзивном подходе решение, полученное с помощью лоренц-ковариантной интерпретации теории функций

\footnotetext{
1)Если, конечно, в силу тех или иных причин корреляции между частицами отсутствуют. Но такое предположение нам представляется маловероятным.
} 
Вигнера [8], [9]. Это дает теоретическую возможность одновременно зафиксировать канонически сопряженную пару переменных, т.е. перейти к теоретическому пределу $\delta R=0$.

Обычные периодические граничные условия Кубо-Мартина-Швингера (КМШ) [10] не применимы к неравновесному случаю [11] (см. также работу [6]). Поэтому, чтобы учесть окружение системы, мы введем граничное условие "руками". Предполагая, что система находится в вакууме, получим стандартное теоретико-полевое вакуумное граничное условие (см. раздел 2). Мы также рассмотрим систему в фоновом поле излучения абсолютно черного тела и покажем, что в пределе равновесия восстанавливается теория с граничными условиями КМШ, т.е. схема канонической термодинамики.

\section{2. ВАКУУМНЫЕ ГРАНИЧНЫЕ УСЛОВИЯ}

Вероятность $r(P)$-перехода из in- в out-состояние при фиксированном полном 4 -импульсе $P$ можно записать с помощью амплитуды $a_{n, m}$ перехода $n$-частичного состояния в $m$-частичное [7]:

$$
a_{n, m}\left((q)_{n},(p)_{m}\right)=\left.\prod_{k=1}^{n} \hat{\phi}\left(q_{k}\right) \prod_{k=1}^{m} \hat{\phi}^{*}\left(p_{k}\right) Z(\phi)\right|_{\phi=0},
$$

где $q_{k}\left(p_{k}\right)$ - импульсы входящих (исходящих) частиц, а также используются операторы уничтожения

$$
\hat{\phi}(q)=\int d^{4} x e^{-i q x} \hat{\phi}(x), \quad \hat{\phi}=\frac{\delta}{\delta \phi(x)},
$$

и соответственно операторы рождения $\hat{\phi}^{*}(p)$. Вспомогательные поля $\phi(x)$ следует положить равными нулю в конце всех вычислений. Амплитуда перехода вакуума в вакуум в присутствии внешнего поля $\phi$

$$
Z(\phi)=\int D \Phi e^{i S_{C_{+}}(\Phi)-i V_{C_{+}}(\Phi+\phi)}
$$

определена на комплексном временно́м контуре Миллса $C_{f}$ [8], т.е. $C_{f}: t \rightarrow t+i \varepsilon$, $\varepsilon>0$. В выражении (2.3) $S_{C_{f}}-$ свободная часть действия, а член $V_{C_{f}}$ описывает взаимодействие.

В этом разделе будет рассмотрено вакуумное граничное условие

$$
\int_{\sigma_{\infty}} d \sigma_{\mu} \Phi \partial^{\mu} \Phi=0
$$

где $\sigma_{\infty}-$ бесконечно удаленная гиперповерхность.

Рассмотрим теперь (ненормированную) вероятность

$$
\begin{array}{rl}
r(P)=\sum_{n, m} & r(P ; n, m)=\sum_{n, m} \frac{1}{n ! m !} \int d \omega_{n}(q) \omega_{m}(p) \times \\
& \times \delta^{(4)}\left(P-\sum_{k=1}^{n} q_{k}\right) \delta^{(4)}\left(P-\sum_{k=1}^{m} p_{k}\right)\left|a_{n, m}\right|^{2},
\end{array}
$$


где

$$
d \omega_{n}(q)=\prod_{k=1}^{n} d \omega\left(q_{k}\right)=\prod_{k=1}^{n} \frac{d^{3} q_{k}}{(2 \pi)^{3} 2 \varepsilon\left(q_{k}\right)}, \quad \varepsilon(q)=\left(q^{2}+m^{2}\right)^{1 / 2} .
$$

Выражение (2.5) является основным для наших выкладок. Соответствующее микроканоническое описание было введено в работе [11] с помощью преобразования Фурье $\delta$-функций законов сохранения в (2.5).

Начнем наше рассмотрение с предположения, что флуктуации температуры имеют большой масштаб. Тогда мы можем предполагать, что в ячейке, размер которой намного меньше масштаба флуктуаций, температура является "хорошим" параметром (в том смысле, что соответствующие флуктуации имеют гауссов вид).

Окружим зону взаимодействия, т.е. интересующую нас систему, $N$ ячейками с известным пространственно-временны́м расположением и предположим, что мы можем измерять энергию и импульс групп входящих и исходящих частиц в каждой ячейке. Четырехмерные ячейки не могут быть произвольно малыми из-за квантового принципа неопределенности.

Разложим $\delta$-функции в выражении $(2.5)$ в виде произведения $(N+1) \delta$-функций:

$$
\delta\left(P-\sum_{k=1}^{n} q_{k}\right)=\int \prod_{\nu=1}^{N}\left\{d Q_{\nu} \delta\left(Q_{\nu}-\sum_{k=1}^{n_{\nu}} q_{k, \nu}\right)\right\} \delta\left(P-\sum_{\nu=1}^{N} Q_{\nu}\right)
$$

где $q_{k, \nu}-$ импульс $k$-й входящей частицы в $\nu$-й ячейке и $Q_{\nu}-$ полный 4 -импульс $n_{\nu}$ входящих частиц в данной ячейке. Такое же разложение будет использовано для второй $\delta$-функции в выражении (2.5). Подставив эти разложения в (2.5), нужно также учесть тождественность частиц. Это даст коэффициент

$$
\frac{n !}{n_{1} ! \ldots n_{N} !} \delta_{\mathrm{K}}\left(n-\sum_{\nu=1}^{N} n_{\nu}\right) \frac{m !}{m_{1} ! \ldots m_{N} !} \delta_{\mathrm{K}}\left(m-\sum_{\nu=1}^{N} m_{\nu}\right)
$$

где $\delta_{\mathrm{K}}-$ символ Кронекера.

В результате выражение

$$
\begin{aligned}
& r\left((Q)_{N},(P)_{N}\right)=\sum_{(n, m)} \int\left|a_{(n, m)}\right|^{2} \times \\
& \quad \times \prod_{\nu=1}^{N}\left\{\prod_{k=1}^{n_{\nu}} \frac{d \omega\left(q_{k, \nu}\right)}{n_{\nu} !} \delta\left(Q_{\nu}-\sum_{k=1}^{n_{\nu}} q_{k, \nu}\right) \prod_{k=1}^{m_{\nu}} \frac{d \omega\left(p_{k, \nu}\right)}{m_{\nu} !} \delta\left(P_{\nu}-\sum_{k=1}^{m_{\nu}} p_{k, \nu}\right)\right\}
\end{aligned}
$$

описывает вероятность наблюдения в $\nu$-й ячейке потоков входящих частиц с полным 4 -импульсом $Q_{\nu}$ и исходящих частиц с полным 4-импульсом $P_{\nu}$ [5]. Последовательность этих двух измерений произвольна.

Преобразование Фурье $\delta$-функций в выражении (2.9) дает

$$
r\left((Q)_{N},(P)_{N}\right)=\int \prod_{\nu=1}^{N} \frac{d^{4} \alpha_{i, \nu}}{(2 \pi)^{4}} \frac{d^{4} \alpha_{f, \nu}}{(2 \pi)^{4}} e^{i \sum_{\nu=1}^{N}\left(Q_{\nu} \alpha_{i, \nu}+P_{\nu} \alpha_{f, \nu}\right)} R\left(\left(\alpha_{i}\right)_{N},\left(\alpha_{f}\right)_{N}\right),
$$


где $R\left(\left(\alpha_{i}\right)_{N},\left(\alpha_{f}\right)_{N}\right) \equiv R\left(\alpha_{i, 1}, \alpha_{i, 2}, \ldots, \alpha_{i, N} ; \alpha_{f, 1}, \alpha_{f, 2}, \ldots, \alpha_{f, N}\right)$ имеет вид

$$
R\left(\left(\alpha_{i}\right)_{N},\left(\alpha_{f}\right)_{N}\right)=\int \prod_{\nu=1}^{N}\left\{\prod_{k=1}^{n_{\nu}} \frac{d \omega\left(q_{k, \nu}\right)}{n_{\nu} !} e^{-i \alpha_{i, \nu} q_{k, \nu}} \prod_{k=1}^{m_{\nu}} \frac{d \omega\left(p_{k, \nu}\right)}{m_{\nu} !} e^{-i \alpha_{f, \nu} p_{k, \nu}}\right\}\left|a_{(n, m)}\right|^{2} .
$$

Подставляя формулу (2.1) в (2.11), получим

$$
\begin{aligned}
R\left(\left(\alpha_{i}\right)_{N},\left(\alpha_{f}\right)_{N}\right)= & \exp \left\{-i \sum_{\nu=1}^{N} \int d x d x^{\prime}\left[\hat{\phi}_{f}(x) D_{f i}\left(x-x^{\prime} ; \alpha_{f, \nu}\right) \hat{\phi}_{i}\left(x^{\prime}\right)-\right.\right. \\
& \left.\left.-\hat{\phi}_{i}(x) D_{i f}\left(x-x^{\prime} ; \alpha_{i, \nu}\right) \hat{\phi}_{f}\left(x^{\prime}\right)\right]\right\} Z\left(\phi_{f}\right) Z^{*}\left(\phi_{i}\right),
\end{aligned}
$$

где поле $\phi_{i}$ определено на комплексно-сопряженном контуре $C_{i}: t \rightarrow t-i \varepsilon$, а также введены положительно- и отрицательно-частотные корреляционные функции

$$
\begin{aligned}
& D_{f i}\left(x-x^{\prime} ; \alpha\right)=-i \int d \omega(q) e^{i q\left(x-x^{\prime}\right)} e^{-i \alpha q}, \\
& D_{i f}\left(x-x^{\prime} ; \alpha\right)=i \int d \omega(q) e^{-i q\left(x-x^{\prime}\right)} e^{-i \alpha q} .
\end{aligned}
$$

При неизвестном распределении потоков импульсов нужно произвести интегрирование по наборам $(Q)_{N}$ и $(P)_{N}$. Тогда

$$
r(P)=\int D^{4} \alpha_{i}(P) D^{4} \alpha_{f}(P) R\left(\left(\alpha_{i}\right)_{N},\left(\alpha_{f}\right)_{N}\right),
$$

где в дифференциальную меру

$$
D^{4} \alpha(P)=\prod_{\nu=1}^{N} \frac{d^{4} \alpha_{\nu}}{(2 \pi)^{4}} K\left(P,(\alpha)_{N}\right)
$$

включен закон сохранения энергии-импульса

$$
K\left(P,(\alpha)_{N}\right)=\int \prod_{\nu=1}^{N} d^{4} Q_{\nu} e^{i \sum_{\nu=1}^{N} \alpha_{\nu} Q_{\nu}} \delta\left(P-\sum_{\nu=1}^{N} Q_{\nu}\right) .
$$

Непосредственное интегрирование дает

$$
K\left(P,(\alpha)_{N}\right) \sim \prod_{\nu=1}^{N} \delta^{(3)}\left(\alpha-\alpha_{\nu}\right)
$$

Для упрощения рассмотрения положим $\alpha=(-i \beta, \overrightarrow{0})$; тогда

$$
K\left(E,(\beta)_{N}\right)=\int_{0}^{\infty} \prod_{\nu=1}^{N} d E_{\nu} e^{\sum_{\nu=1}^{N} \beta_{\nu} E_{\nu}} \delta\left(E-\sum_{\nu=1}^{N} E_{\nu}\right)
$$

Делая явную подстановку $\alpha_{k, \nu}=\left(-i \beta_{k, \nu}, \overrightarrow{0}\right), \operatorname{Re} \beta_{k, \nu}>0, k=f, i$, получим

$$
r(E)=\int D \beta_{f}(E) D \beta_{i}(E) R\left(\left(\beta_{f}\right)_{N},\left(\beta_{i}\right)_{N}\right),
$$


где

$$
D \beta(E)=\prod_{\nu=1}^{N} \frac{d \beta_{\nu}}{2 \pi i} K\left(E,(\beta)_{N}\right)
$$

и $R\left((\beta)_{N}\right)$ определено в $(2.12)$.

Интеграл по $\beta_{k}$ найдем с помощью метода стационарной фазы. Уравнение для наиболее вероятного значения $\beta_{k}$

$$
-\frac{1}{K\left(E,\left(\beta_{k}\right)_{N}\right)} \frac{\partial}{\partial \beta_{k, \nu}} K\left(E,\left(\beta_{k}\right)_{N}\right)=\frac{1}{R\left(\left(\beta_{1}\right)_{N}\right)} \frac{\partial}{\partial \beta_{k, \nu}} R\left((\beta)_{N}\right), \quad k=f, i,
$$

всегда имеет единственное положительное решение $\tilde{\beta}_{k, \nu}(E)$. При этом мы предполагаем, что отклонения $\beta_{k}$ от наиболее вероятного значения $\tilde{\beta}_{k}$ малы, т.е. являются гауссовыми. Это основа гипотезы локального равновесия [7]. В этом случае $1 / \tilde{\beta}_{i, \nu}-$ температура начального состояния, а $1 / \tilde{\beta}_{f, \nu}$ - температура конечного состояния в $\nu$-й измерительной ячейке.

Формулировка (2.15) подразумевает, что 4-импульсы $(Q)_{N}$ и $(P)_{N}$ не могут быть измерены. Можно рассмотреть другую интерпретацию. Например, предположим, что начальный набор $(Q)_{N}$ зафиксирован (измерен), а набор $(P)_{N}-$ нет. В этом случае мы имеем смешанный эксперимент: $\tilde{\beta}_{i, \nu}$ определяется из уравнения

$$
E_{\nu}=-\frac{1}{R} \frac{\partial}{\partial \beta_{i, \nu}} R
$$

а $\tilde{\beta}_{f, \nu}-$ из уравнения $(2.22)$ при $k=f$.

В пределе $N \rightarrow \infty$ размер ячеек стремится к нулю. В этом случае квантовый принцип неопределенности вынуждает нас считать, что наборы 4-импульсов $(Q)$ и $(P)$ не фиксируются. Предполагая, что $\left(\beta_{i}\right)$ и $\left(\beta_{f}\right)$ - измеримые величины, мы приходим к чистой термодинамике. Например, можно зафиксировать $\left(\beta_{i}\right)$ и найти $\left(\beta_{f}\right)$ как функцию от $E$ и $\left(\beta_{i}\right)$. Тогда уравнение $(2.22)$ становится функциональным.

В рассмотренном микроканоническом описании введение температурного описания не влияет на схему квантования. Действительно, как это следует из выражения (2.12), вся термодинамическая информация содержится в операторной экспоненте

$$
\prod_{\nu} \prod_{i \neq j} e^{i \int \hat{\phi}_{i} D_{i j} \hat{\phi}_{j}}=e^{N\left(\hat{\phi}_{i} \hat{\phi}_{j}\right)},
$$

наличие которой определяет "внешние" (наблюдаемые) условия, а "внутренние” возмущения описываются амплитудой $Z(\phi)$. Такая факторизация стала возможной изза введения вспомогательного поля $\phi$ и не зависит от выбора граничных условий, т.е. от "внешних" условий.

\section{3. ФУНКЦИИ РАСПРЕДЕЛЕНИЯ}

В предыдущем разделе производящий функционал $R\left((\beta)_{N}\right)$ был вычислен с помощью разделения "измерительного устройства" (калориметра) на $N$ ячеек в предположении, что их размер стремится к нулю $(N \rightarrow \infty)$. Теперь определим координаты этих ячеек, используя формализм Вигнера [6], [9]. 
Рассмотрим функцию распределения $F_{n}$, определяющую вероятность нахождения $n$ частиц с определенными импульсами и произвольными координатами. Эта вероятность (сечение рассеяния) обычно измеряется в физике частиц. Соответствующий фурье-преобразованный производящий функционал можно получить из выражения (2.12):

$$
F\left(z,\left(\beta_{f}\right)_{N},\left(\beta_{i}\right)_{N}\right)=\prod_{\nu=1}^{N} \prod_{k \neq j} \exp \left\{\int d \omega(q) \hat{\phi}_{k}^{*}(q) e^{-\beta_{j, \nu} \varepsilon(q)} \hat{\phi}_{j}(q) z_{k j}^{\nu}(q)\right\} Z\left(\phi_{f}\right) Z^{*}\left(\phi_{i}\right) .
$$

Вариация $F$ по $z_{k j}^{\nu}(q)$ дает соответствующую функцию распределения. При этом $z_{k j}^{\nu}(q)$ можно интерпретировать как локальную активность: логарифм от $z_{k j}^{\nu}(q)$ канонически сопряжен числу частиц с импульсом $q$ в $\nu$-й ячейке для начального $(k j=f i)$ или конечного $(k j=i f)$ состояний, а выражение $z_{k j}^{\nu}(q) \hat{\phi}_{k}^{*}(q) \hat{\phi}_{j}(q)$ можно рассматривать как оператор активности.

Больцмановский фактор $e^{-\beta_{j, \nu} \varepsilon(q)}$ можно интерпретировать как вероятность нахождения частицы с энергией $\varepsilon(q)$ в конечном $(j=f)$ или начальном $(j=i)$ состояниях. Полная вероятность процесса рождения и дальнейшего уничтожения $n$ частиц равна соответственно произведению этих факторов.

Производящий функционал (3.1) нормируется следующим образом:

$$
\begin{gathered}
F(z=1,(\beta))=R((\beta)), \\
F(z=0,(\beta))=|Z(0)|^{2}=\left.R_{0}(\phi)\right|_{\phi=0},
\end{gathered}
$$

где

$$
R_{0}(\phi)=Z\left(\phi_{f}\right) Z^{*}\left(\phi_{i}\right)
$$

- "вероятность" перехода вакуума в вакуум в присутствии вспомогательного поля $\phi_{i(f)}$. Одночастичная функция распределения

$$
F_{1}\left(\left(\beta_{f}\right)_{N},\left(\beta_{i}\right)_{N} ; q\right)=\left.\frac{\delta}{\delta z_{k j}^{\nu}(q)} F\right|_{z=0}=\left\{\hat{\phi}_{k}^{*}(q) e^{-\beta_{k}^{\nu} \varepsilon(q) / 2}\right\}\left\{\hat{\phi}_{j}(q) e^{-\beta_{k}^{\nu} \varepsilon(q) / 2}\right\} R_{0}(\phi)
$$

описывает вероятность нахождения одной частицы с импульсом $q$ в $\nu$-й ячейке.

Используя определение (2.2), перепишем функцию распределения в виде

$$
\begin{aligned}
F_{1}\left(\left(\beta_{f}\right)_{N},\left(\beta_{i}\right)_{N} ; q\right) & =\int d x d x^{\prime} e^{i q\left(x-x^{\prime}\right)} e^{-\beta_{k, \nu} \varepsilon(q)} \hat{\phi}_{k}(x) \hat{\phi}_{j}\left(x^{\prime}\right) R_{0}(\phi)= \\
& =\int d Y\left\{d y e^{i q y} e^{-\beta_{i, \nu} \varepsilon(q)}\right\} \hat{\phi}_{i}\left(Y+\frac{y}{2}\right) \hat{\phi}_{j}\left(Y-\frac{y}{2}\right) R_{0}(\phi) .
\end{aligned}
$$

С помощью этого выражения можно определить “одночастичную функцию Вигнеpa" $W_{1}[8]$, [9]:

$$
F_{1}\left(\left(\beta_{f}\right)_{N},\left(\beta_{i}\right)_{N} ; q\right)=\int d Y W_{1}\left(\left(\beta_{f}\right)_{N},\left(\beta_{i}\right)_{N} ; Y, q\right)
$$

где

$$
W_{1}\left(\left(\beta_{f}\right)_{N},\left(\beta_{i}\right)_{N} ; Y, q\right)=\int d y e^{i q y} e^{-\beta_{k, \nu} \varepsilon(q)} \hat{\phi}_{k}\left(Y+\frac{y}{2}\right) \hat{\phi}_{j}\left(Y-\frac{y}{2}\right) R_{0}(\phi) .
$$


Функция распределения $W_{1}$ описывает условную вероятность нахождения свободной частицы с импульсом $q$ в точке $Y$ в $\nu$-й ячейке.

Поскольку координаты устройства мы выбираем произвольно, удобно соотнести их с координатой измерения $Y$ :

$$
W_{1}\left(\left(\beta_{f}\right)_{N},\left(\beta_{i}\right)_{N} ; Y, q\right)=\int d y e^{i q y} e^{-\beta_{k}(Y) \varepsilon(q)} \hat{\phi}_{k}\left(Y+\frac{y}{2}\right) \hat{\phi}_{j}\left(Y-\frac{y}{2}\right) R_{0}(\phi) .
$$

Чтобы сделать более ясным физический смысл координат фазового пространства $(Y, q)$, в приложении выводится уравнение Лиувиля для $W_{1}$.

Такой выбор координат прибора приводит к следующему виду производящего функционала:

$$
\begin{gathered}
F(z, \beta)=\exp \left\{i \int d y d Y \left[\hat{\phi}_{f}\left(Y+\frac{y}{2}\right) D_{f i}\left(y ; \beta_{f}(Y), z\right) \hat{\phi}_{i}\left(Y-\frac{y}{2}\right)-\right.\right. \\
\left.\left.-\hat{\phi}_{i}\left(Y+\frac{y}{2}\right) D_{i f}\left(y ; \beta_{i}(Y), z\right) \hat{\phi}_{f}\left(Y-\frac{y}{2}\right)\right]\right\} R_{0}(\phi),
\end{gathered}
$$

где

$$
\begin{aligned}
& D_{f i}\left(y ; \beta_{f}(Y), z\right)=-i \int d \omega(q) z_{f i}(Y, q) e^{i q y} e^{-\beta_{f}(Y) \varepsilon(q)}, \\
& D_{i f}\left(y ; \beta_{f}(Y), z\right)=i \int d \omega(q) z_{i f}(Y, q) e^{-i q y} e^{-\beta_{i}(Y) \varepsilon(q)}
\end{aligned}
$$

- модифицированные положительно- и отрицательно-частотные корреляционные функции (2.13), (2.14).

\section{4. ПЕРИОДИЧЕСКИЕ ГРАНИЧНЫЕ УСЛОВИЯ}

Развитый в разделе 2 формализм можно рассмотреть при более общих, чем (2.3), граничных условиях. Представление вероятности $R$ в виде двойного функционального интеграла позволяет ввести интегрирование по замкнутому контуру и, следовательно, рассматривать равенство

$$
\int_{\sigma_{\infty}} d \sigma_{\mu}\left(\Phi_{f} \partial^{\mu} \Phi_{f}-\Phi_{i} \partial^{\mu} \Phi_{i}\right)=0
$$

как граничное условие, где $\sigma_{\infty}-$ бесконечно удаленная гиперповерхность. Решение этого уравнения выглядит, например, следующим образом:

$$
\Phi_{i}\left(\sigma_{\infty}\right)=\Phi_{f}\left(\sigma_{\infty}\right)=\Phi\left(\sigma_{\infty}\right),
$$

где $\sigma_{\infty}$ - “точки разворота" траектории. Такая замена граничных условий для случая однородного распределения температуры была проанализирована в работе [11].

В терминах $S$-матрицы поле $\Phi\left(\sigma_{\infty}\right)$ представляется как фоновое поле частиц на массовой поверхности. Мы будем считать, что вероятность нахождения частицы в фоновом потоке определяется только законом сохранения энергии-импульса. Другими словами, будем считать, что рассматриваемая нами система окружена излучением черного тела. 
Наличие дополнительного потока невзаимодействующих частиц переопределяет только дифференциальный оператор $e^{N\left(\hat{\phi}_{i} \hat{\phi}_{j}\right)}$. Поэтому новый производящий функционал по замкнутому пути $R_{\bigcirc}$ имеет вид

$$
R_{\bigcirc}\left(\alpha_{f}, \alpha_{i}\right)=e^{N\left(\hat{\phi}_{i} \hat{\phi}_{j}\right)} R_{0}(\phi)
$$

Найдем оператор $N\left(\hat{\phi}_{i} \hat{\phi}_{j}\right)$ точно так же, как это было сделано в работе [11]. Вводя ячейки по $Y$-пространству, получим

$$
N\left(\hat{\phi}_{i} \hat{\phi}_{j}\right)=\int d Y d y \hat{\phi}_{i}\left(Y+\frac{y}{2}\right) \tilde{n}_{i j}(Y, y) \hat{\phi}_{j}\left(Y-\frac{y}{2}\right),
$$

где числа заполнения $\tilde{n}_{i j}$ несут индекс ячейки $Y$ :

$$
\begin{gathered}
\tilde{n}_{i j}(Y, y)=\int d \omega(q) e^{i q y} n_{i j}(Y, q), \quad q_{0}=\varepsilon(q), \\
n_{f f}\left(Y, q_{0}\right)=n_{i i}\left(Y, q_{0}\right)=\tilde{n}\left(Y, \frac{\left(\beta_{f}+\beta_{i}\right)\left|q_{0}\right|}{2}\right)=\frac{1}{e^{\left(\beta_{f}+\beta_{i}\right)(Y)\left|q_{0}\right| / 2}-1}, \\
n_{f i}\left(Y, q_{0}\right)=\Theta\left(q_{0}\right)\left(1+\tilde{n}\left(Y, \beta_{f} q_{0}\right)\right)+\Theta\left(-q_{0}\right) \tilde{n}\left(Y,-\beta_{i} q_{0}\right), \\
n_{i f}\left(Y, q_{0}\right)=n_{f i}\left(Y,-q_{0}\right) .
\end{gathered}
$$

Здесь для простоты использовалась система центра масс.

Вычисляя $R_{0}$ по теории возмущений, получим

$$
\begin{aligned}
R_{\bigcirc}(\beta)=\exp \left\{-i V\left(-i \hat{j}_{f}\right)+i V\left(-i \hat{j}_{i}\right)\right\} \times \\
\times \exp \left\{i \int d Y d y\left[j_{i}\left(Y+\frac{y}{2}\right) G_{i j}(y,(\beta(Y))) j_{j}\left(Y-\frac{y}{2}\right)\right]\right\},
\end{aligned}
$$

где в матричном представлении

$$
\begin{aligned}
i G(q,(\beta(Y)))= & \left(\begin{array}{cc}
\frac{i}{q^{2}-m^{2}+i \hat{e}} & 0 \\
0 & -\frac{i}{q^{2}-m^{2}-i \hat{e}}
\end{array}\right)+ \\
& +2 \pi \delta\left(q^{2}-m^{2}\right)\left(\begin{array}{cc}
n\left(\frac{\left(\beta_{f}+\beta_{i}\right)(Y)}{2}\left|q_{0}\right|\right) & n\left(\beta_{f}(Y)\left|q_{0}\right|\right) a_{f}\left(\beta_{f}\right) \\
n\left(\beta_{i}(Y)\left|q_{0}\right|\right) a_{i}\left(\beta_{i}\right) & n\left(\frac{\left(\beta_{f}+\beta_{i}\right)(Y)}{2}\left|q_{0}\right|\right)
\end{array}\right)
\end{aligned}
$$

и

$$
a_{f}\left(\beta_{f}\right)=-e^{\beta_{f}\left(\left|q_{0}\right|+q_{0}\right) / 2}, \quad a_{i}\left(\beta_{i}\right)=-e^{\beta_{i}\left(\left|q_{0}\right|-q_{0}\right) / 2} .
$$

Формально эта функция Грина удовлетворяет стандартным уравнениям в пространстве $y$ :

$$
\begin{aligned}
& \left(\partial^{2}-m^{2}\right)_{y} G_{i i}=\delta(y), \\
& \left(\partial^{2}-m^{2}\right)_{y} G_{i j}=0, \quad i \neq j,
\end{aligned}
$$

поскольку $\Phi\left(\sigma_{\infty}\right) \neq 0$ означает наличие частиц на массовой поверхности. 


\section{5. ЗАКЛЮЧИТЕЛЬНЫЕ ЗАМЕЧАНИЯ}

Естественное обобщение инклюзивного подхода Логунова состоит в предложении рассматривать пучок частиц в одной ячейке как одну частицу. В нашем случае эта идея основана на возможности того, что подсистемы частиц могут находиться в "предравновесном" состоянии.

Иными словами, можно полагать, что "предравновесная" среда состоит из макроскопического размера равновесных доменов $\Omega(Y)$. Мы предлагаем рассматривать подсистемы частиц $\Omega_{q}(Y) \in \Omega(Y)$ как частицу с координатой $Y$. Такая подсистема может иметь какое-то дополнительное свойство $q$, например, температура всех частиц подсистемы может быть одинаковой.

Другим интересным вопросом является возможность описания фазового пространства состояний рождающихся при столкновениях частиц с высокой энергией. Релятивистская функция распределения фазового пространства может быть построена с помощью функции Вигнера $W_{1}$ (см. (3.7)). Это следует из того факта, что $W_{1}$ подчиняется уравнению Лиувилля, т.е. объем фазового пространства сохраняется, что легко доказать, основываясь на представлении функционального интеграла (3.9) (см. приложение).

\section{ПРИЛОЖЕНИЕ}

\section{Уравнение Лиувилля для функции Вигнера}

Рассмотрим функциональный интеграл (3.9) и исследуем, при каких условиях функция $W_{1}$ подчиняется уравнению Лиувилля.

Запишем $W_{1}$ в виде

$$
\begin{aligned}
W_{1}(\beta ; Y, q) & =\int d y e^{i q y} e^{-\beta_{k}(Y) \varepsilon(q)} \hat{\phi}_{k}\left(Y+\frac{y}{2}\right) \hat{\phi}_{j}\left(Y-\frac{y}{2}\right) \times \\
& \times \int D \Phi_{+} D \Phi_{-} e^{i S_{C_{+}\left(t_{\mathrm{in}}\right)}^{0}\left(\Phi_{+}\right)-i V_{C_{+}\left(t_{\mathrm{in}}\right)}\left(\Phi_{+}+\phi_{f}\right)-i S_{C_{-}\left(t_{\mathrm{in}}\right)}^{0}\left(\Phi_{-}\right)+i V_{C_{-}\left(t_{\mathrm{in}}\right)}\left(\Phi_{-}-\phi_{i}\right)}= \\
= & \int d y e^{i q y} e^{-\beta_{k}(Y) \varepsilon(q)} \int D \Phi_{+} D \Phi_{-} V_{C_{+}\left(t_{\mathrm{in}}\right)}^{\prime}\left(\Phi_{+} ; Y+\frac{y}{2}\right) \times \\
\times & V_{C_{-}\left(t_{\mathrm{in}}\right)}^{\prime}\left(\Phi_{-} ; Y-\frac{y}{2}\right) e^{i S_{C_{+}\left(t_{\mathrm{in}}\right)}\left(\Phi_{+}\right)-i S_{C_{-}\left(t_{\mathrm{in}}\right)}\left(\Phi_{-}\right)}
\end{aligned}
$$

где $S_{C_{ \pm}\left(t_{\mathrm{in}}\right)}^{0}-$ свободная часть полного действия $S_{C_{ \pm}\left(t_{\mathrm{in}}\right)}\left(\Phi_{ \pm}\right)=S_{C_{ \pm}\left(t_{\mathrm{in}}\right)}^{0}\left(\Phi_{ \pm}\right)-$ $V_{C_{ \pm}\left(t_{\mathrm{in}}\right)}\left(\Phi_{ \pm}\right)$, определенного на комплексном временно́м контуре Миллса:

$$
C_{ \pm}\left(t_{\text {in }}\right): t \rightarrow \pm i \epsilon, \quad \epsilon \rightarrow+0, \quad t_{\text {in }} \leqslant t \leqslant+\infty, \quad t_{\text {in }} \rightarrow-\infty .
$$

Мы будем использовать замкнутое граничное условие (4.2):

$$
\Phi_{ \pm}\left(\sigma_{t_{\text {in }}}\right)=\Phi\left(\sigma_{t_{\text {in }}}\right)
$$

где гиперповерхность $\sigma_{t_{\text {in }}}$ проходит через точку $t_{\text {in }}$. Следует подчеркнуть, что по "точкам разворота" поля $\Phi\left(\sigma_{t_{\mathrm{in}}}\right)$ нужно проинтегрировать. Замкнутые граничные 
условия следует брать при конечных $t_{\text {in }}$, для того чтобы сохранить их отличными от поверхностных членов.

Представление (П.1) содержит вершины

$$
V_{C_{-}\left(t_{\mathrm{in}}\right)}^{\prime}\left(\Phi_{ \pm} ; Y \pm \frac{y}{2}\right)=\frac{\delta}{\delta \Phi_{ \pm}(Y \pm y / 2)} V_{C_{-}\left(t_{\mathrm{in}}\right)}\left(\Phi_{ \pm}\right) .
$$

Заметим, что нет необходимости обрезать интеграл по $y$ в точке $y=t_{\text {in }}$, поскольку действие оператора $\hat{\phi}_{j}(Y \pm y / 2)$ заканчивается в момент времени $(Y \pm y / 2)_{0}=t_{\text {in }}$. Поэтому $W_{1}(\beta ; Y, q)$ существует для временно́го интервала $Y_{0}<t_{\text {in }}$. Зависимость $D \Phi_{ \pm}$от $t_{\text {in }}$ несущественна, поскольку мы всегда можем добавить (бесконечное) интегрирование $\prod_{t=-\infty}^{t_{\text {in }}} d \Phi(t)$, понимая, что этот бесконечный вклад сокращается нормировочным множителем.

Как было показано ранее, двойной функциональный интеграл (П.1) определен на $\delta$-образной мере Дирака [6]. Результат выглядит следующим образом:

$$
\begin{aligned}
W_{1}(\beta ; Y, q)=\int & d y e^{i q y} e^{-\beta_{k}(Y) \varepsilon(q)} e^{-i \mathbf{K}(j e)} \times \\
& \times \int D M(\Phi) V_{C_{+}\left(t_{\text {in }}\right)}^{\prime}\left(\Phi+e ; Y+\frac{y}{2}\right) V_{C_{-}\left(t_{\text {in }}\right)}^{\prime}\left(\Phi-e ; Y-\frac{y}{2}\right) e^{-i U_{C}(\Phi ; e)} .
\end{aligned}
$$

Разложение экспоненты $e^{-i \mathbf{K}(j e)}$ в ряд по степеням оператора

$$
2 \mathbf{K}(j e)=\int d x \frac{\delta}{\delta j(x)} \frac{\delta}{\delta e(x)}
$$

дает обычную теорию возмущений. Заметим, что оператор $\mathbf{K}(j e)$ не зависит от $t_{\text {in }}$.

Функциональный интеграл (П.5) определен на мере Дирака

$$
D M(\Phi)=\prod_{x}^{\prime} d \Phi(x) \delta\left(\frac{\delta S(\Phi)}{\delta \Phi(x)}-j(x)\right)
$$

где штрих означает, что функциональные $\delta$-функции не содержат точку $x_{0}=t_{\text {in }}$.

Функционал $U_{C_{+}}(\Phi ; \phi, e)$ описывает взаимодействие [6]. Явный его вид, вообще говоря, для нас не важен.

Для вывода уравнения Лиувилля перейдем к фазовому пространству. Легко видеть, что мера (П.7) имеет следующий вид:

$$
D M(\Phi)=\prod_{x} d \Phi(x) d \mathrm{P}(x) \delta\left(\dot{\Phi}-\frac{\delta H_{j}(\Phi, \mathrm{P})}{\delta \mathrm{P}(x)}\right) \delta\left(\dot{\mathrm{P}}+\frac{\delta H_{j}(\Phi, \mathrm{P})}{\delta \Phi(x)}\right),
$$

где гамильтониан

$$
H_{j}(\Phi, \mathrm{P})=\int d^{3} x\left\{\frac{1}{2} \mathrm{P}^{2}+\frac{1}{2}(\nabla \Phi)^{2}+v(\Phi)-j \Phi\right\}
$$

явно зависит от источника $j(x)$, производящего квантовые возмущения, и потенциального члена $v(\Phi)$. Переход от $(П .7)$ к (П.8) может вызвать некоторые сомнения 
из-за возможной симметрии рассматриваемой задачи. Чтобы избежать этой неоднозначности, такой переход можно рассматривать как переход к формализму первого порядка.

Заметим теперь, что равенства

$$
\dot{\Phi}=\frac{\delta H_{j}(\Phi, \mathrm{P})}{\delta \mathrm{P}(x)}, \quad \dot{\mathrm{P}}=-\frac{\delta H_{j}(\Phi, \mathrm{P})}{\delta \Phi(x)}
$$

сохраняются для любых $\Phi_{0}$ и $\mathrm{P}_{0}$. Поэтому можно опустить штрих в мере (П.8), т.е. включить границу $t=t_{\text {in }}$ в меру.

Подчеркнем, что, следуя нашему определению,

$$
\int \prod_{x} d \Phi(x) \delta(\dot{\Phi}(x))=\int d \Phi\left(t_{\mathrm{in}}\right)=\int d \Phi_{0}
$$

Итак, функциональные $\delta$-функции оставляют не определенными граничные значения $\left(\Phi_{0}, \mathrm{P}_{0}\right)$, по которым, вообще говоря, следует интегрировать:

$$
W_{1}\left(\beta ; Y, q, t_{i} n\right)=\int d \Phi_{0} d \mathrm{P}_{0} W_{1}\left(\beta ; Y, q, \Phi_{0}, \mathrm{P}_{0}\right)
$$

Уравнение Лиувилля существует только для $W_{1}\left(\beta ; Y, q, \Phi_{0}, \mathrm{P}_{0}\right)$.

Действительно, найдем полную производную по $t_{\text {in }}$ :

$$
\frac{d}{d t_{\mathrm{in}}} W_{1}\left(\beta ; Y, q, \Phi_{0}, \mathrm{P}_{0}\right)=\frac{\partial W_{1}\left(\beta ; Y, q, \Phi_{0}, \mathrm{P}_{0}\right)}{\partial \Phi_{0}} \dot{\Phi}_{0}+\frac{\partial W_{1}\left(\beta ; Y, q, \Phi_{0}, \mathrm{P}_{0}\right)}{\partial \mathrm{P}_{0}} \dot{\mathrm{P}}_{0} .
$$

Воспользовавшись мерой (П.8), можно написать, что

$$
\frac{d}{d t_{\text {in }}} W_{1}\left(\beta ; Y, q, \Phi_{0}, \mathrm{P}_{0}\right)=e^{-i \mathbf{K}(j e)} e^{-i U_{C}(\Phi ; 0, e)}\left\{W_{1}\left(\beta ; Y, q, \Phi_{0}, \mathrm{P}_{0}\right), H_{j}\left(\Phi_{0}, \mathrm{P}_{0}\right)\right\},
$$

где скобки Пуассона имеют вид

$$
\left\{W_{1}, H_{j}\right\}=\frac{\partial W_{1}}{\partial \Phi} \frac{\partial H_{j}}{\partial \mathrm{P}}-\frac{\partial W_{1}}{\partial \mathrm{P}} \frac{\partial H_{j}}{\partial \Phi}
$$

Заметим, что уравнение (П.13) имеет квантовый характер: правая часть содержит оператор квантовых возмущений $\mathbf{K}(j e)$, действующий на источник $j$, от которого зависит гамильтониан $H_{j}=H_{j}\left(\Phi_{0}, \mathrm{P}_{0}\right)$, и на $e$, от которой зависит функционал $U_{C}(\Phi ; 0, e)$, описывающий взаимодействие. Все величины при этом определены в момент времени $t_{\text {in }}$.

Благодарности. Авторы благодарны В.Г. Кадышевскому, В.А. Матвееву, А. С. Сорину и А.Н. Тавхелидзе за интерес к работе. Работа выполнена при поддержке РФФИ (грант № 05-02-17693). 


\section{Список литературы}

[1] A. A. Logunov, M. A. Mestvirishvili, Nguen van Hieu, Phys. Lett. B, 25 (1967), 611; R. Feynman, Phys. Rev. Lett., 23 (1969), 1415.

[2] A. A. Logunov, M. A. Mestvirishvili, V. A. Petrov, Ann. Phys., 114 (1978), 46.

[3] А.А. Логунов, М. А. Мествиришвили, Нгуен Ван Хьеу, Может ли быть локальным эффективное взаимодействие адронов в инклюзивных процессах, Препринт ИФВЭ 7466, ИФВЭ, Протвино, 1974.

[4] Н.Н. Боголюбов, Проблемы динамической теории в статистической физике, Гостехиздат, М., 1946.

[5] А. А. Логунов, М. А. Мествиришвили, В. А. Петров, ЭЧАЯ, 14 (1983), 493.

[6] J. Manjavidze, A. Sissakian, Phys. Rep., 346 (2001), 1.

[7] J. Manjavidze, A. Sissakian, Part. \& Nucl., 31 (2000), 104.

[8] E. P. Wigner, Phys. Rev., 40 (1932), 749.

[9] E. Carruthers, F. Zachariasen, Rev. Mod. Phys., 55 (1983), 245; E. Calsetta, B. L. Hu, Phys. Rev. D, 37 (1988), 2878.

[10] P. S. Martin, J. Schwinger, Phys. Rev., 115 (1959), 1342.

[11] T. Bibilashvili, I. Pasiashvili, Ann. Phys., 220 (1992), 134; T. Bibilashvili, Phys. Lett. B, 313 (1993), 119. 